\title{
Nonlinear Microwave Characterization of CVD Grown Graphene
}

\author{
Mingguang Tuo, Dongchao Xu, Si Li, Min Liang, Student Member, IEEE, Qi Zhu, Qing Hao, and Hao \\ Xin, Senior Member, IEEE
}

\begin{abstract}
Linear and nonlinear microwave properties of chemical vapor deposition (CVD) grown graphene are characterized by incorporating a co-planar waveguide (CPW) transmission line test structure. The intrinsic linear transport properties ( $S$-parameters) of the graphene sample are measured and extracted via a de-embedding procedure and then fitted with an equivalent circuit model up to $10 \mathrm{GHz}$. A statistical uncertainty analysis based on multiple measurements is implemented to estimate the error of the extracted graphene linear parameters as well. Nonlinear properties (second- and third-order harmonics as a function of fundamental input power) of the sample are also measured with a fundamental input signal of 1 GHz. Clear harmonics generated from graphene are observed while no obvious fundamental power saturation is seen. The measured nonlinearity is applied in a graphene patch antenna case study to understand its influence on potential applications in terms of third-order intermodulation levels.
\end{abstract}

Index Terms-Equivalent Circuit Model, Graphene, Intermodulation, Nonlinearity.

\section{INTRODUCTION}

A $\mathrm{S}$ a two-dimensional single layer of carbon atoms, graphene has attracted tremendous interests due to its excellent electrical, mechanical and thermal properties. Many high-frequency applications, including meta-materials, absorbers, and antennas [1-4] have been proposed to utilize those advantages. With the help of chemical vapor deposition (CVD) technique, large-scale production of graphene layers also becomes possible. Therefore, thorough experimental study and understanding of high-frequency transport properties of CVD grown graphene is necessary to enable potential applications at microwave or even $\mathrm{THz}$ frequency regime.

Surface conductivity of graphene is a fundamental parameter which is useful in the designs for various graphene

Mingguang Tuo, Min Liang and Hao Xin are with the Department of Electrical and Computer Engineering, University of Arizona, Tucson, AZ, USA (e-mail: hxin@ece.arizona.edu).

Dongchao $\mathrm{Xu}$ and Hao Qing are with the Department of Aerospace and Mechanical Engineering, University of Arizona, Tucson, AZ, USA (e-mail: qinghao@email.arizona.edu).

$\mathrm{Si} \mathrm{Li}$ is with the Department of Electronic Engineering and Information Science, University of Science and Technology of China, Hefei, Anhui, CHN and the Department of Electrical and Computer Engineering, University of Arizona, Tucson, AZ, USA.

Qi Zhu is with the Department of Electronic Engineering and Information Science, University of Science and Technology of China, Hefei, Anhui, CHN. based components. Although it can be theoretically calculated by Kubo formula [5], it still varies quite much experimentally [6-8] due to sample difference, for example, defects and impurities [9].

In this work, the intrinsic microwave transport properties of CVD grown single-atomic-layer graphene are characterized up to $10 \mathrm{GHz}$ using a $50-\Omega \mathrm{CPW}$ transmission line test structure due to its ease of design and fabrication. An equivalent circuit model is proposed to fit the de-embedded measurement data and the corresponding surface conductivity of the graphene sample is extracted. Previously, a CPW transmission line structure was used to characterize the properties of a graphene sample in [10]. With respect to their work, the nonlinear microwave properties of the graphene sample are studied here. Moreover, the statistical uncertainty of the extracted parameters is analyzed based on a number of repeated measurements with independent calibrations. Those parameters are useful to study the performance of various high-frequency applications such as antennas [11-12].

Strong nonlinear electromagnetic behavior has been predicted theoretically [13]. This results from the linear dispersion of the carriers in graphene, instead of the parabolic dispersion of conventional semiconductors. Some works have reported demonstration of frequency multiplier and mixer at microwave frequency range [14-16]. However, no systematic microwave nonlinear property study on graphene has been reported. Therefore, it is indispensable to further investigate and understand the nonlinear properties of graphene at microwave frequency range for potential applications such as antennas. For example, with two or more signals simultaneously present at the input of a graphene antenna, unwanted third-order intermodulation distortion (IMD3) may occur and therefore limit the performance of a wireless communication system. For commercial antennas, the IMD3 level could be measured as low as $-140 \mathrm{dBc}$, which comes from contact nonlinearity such as loose, oxidized and contaminated metallic joints, with about $+40 \mathrm{dBm}$ carrier input [17]. With the potentially strong nonlinearity in graphene, the IMD3 level could be high to deteriorate practical system performance. Therefore, in this work, nonlinear responses of the CPW graphene sample are experimentally examined under a fundamental input signal of $1 \mathrm{GHz}$ at different power levels up to $+16 \mathrm{dBm}$. Both weak second-order and strong third-order harmonics are observed. The maximum input power to a graphene antenna to avoid 
unacceptable IMD3 level is also estimated.

\section{TEST FIXTURE DESIGN AND FABRICATION}

\section{A. Test Fixture Design}

The test fixture is designed to be on a high-resistivity ( $\rho=$ 20,000 - 40,000 $\Omega \cdot \mathrm{cm}$ ) Si substrate. Previously, a tapered CPW transmission line test fixture is adapted to characterize individual single-walled carbon nanotube (SWCNT) [18]. However, since graphene does not have large intrinsic impedance as SWCNT, a simple $50-\Omega$ CPW transmission line structure with a center gap in the signal trace is designed for characterizing the graphene sample. The schematic and dimensions of the test fixture structure are presented in [19].

\section{B. Test Device Fabrication}

The above designed CPW structure is fabricated on $500-\mu \mathrm{m}$ thick high resistivity $\mathrm{Si}$ substrate $(1 \mathrm{~cm} \times 1 \mathrm{~cm})$ by conventional photo-lithography and lift-off processes. The conductor is made of $15 \mathrm{~nm} \mathrm{Cr} / 100 \mathrm{~nm} \mathrm{Au}$ via e-beam evaporation. The graphene sample is firstly grown by CVD on a copper foil and then transferred onto the CPW structure. The copper foil is initially cleaned with solvents, electrochemically polished and then annealed at a high temperature to remove impurities. The detailed growth condition and structural information of the CVD graphene can be found elsewhere [20]. Since the graphene sample size is larger than the CPW signal-ground spacing, an additional exposure is implemented so that the unwanted graphene is trimmed by plasma cleaning. A sample photo image of the graphene device is also available in [19]. To extract the intrinsic microwave transport properties of the graphene sample, "Open" (no graphene across the center gap) and "Through" (continuous CPW signal trace without the center gap) calibration standards are fabricated on the same high resistivity Si substrate as well for de-embedding purpose.

\section{EXPERIMENTAL CHARACTERIZATION AND ANALYSIS}

\section{A. Linear Characterization}

The linear transport properties ( $S$-parameters) of the graphene test fixture are measured by an Agilent E8361A VNA with the input RF signal set to be $-17 \mathrm{dBm}$. The measurement setup is similar to the one reported in [18]. 150 $\mu \mathrm{m}$-pitch Ground-Signal-Ground (GSG) CPW wafer probes are used to characterize the test device under study. A standard Short-Open-Load-Through (SOLT) on-wafer calibration is done using a calibration substrate from GGB Industries Inc. The graphene device along with the above mentioned "Open" and "Through" structures are measured under the same calibration condition up to $10 \mathrm{GHz}$. The measured $S$-parameters for each structure are plotted in Fig. 1. To obtain the intrinsic properties of the graphene sample, the effects due to the test fixture have to be eliminated. An openthrough de-embedding method [21] is applied and an equivalent circuit model is proposed to fit the de-embedded measurement data. In the equivalent circuit model shown in Fig. $2, R_{\mathrm{C}}$ denotes the contact resistance and $C_{\mathrm{C}}$ is the contact

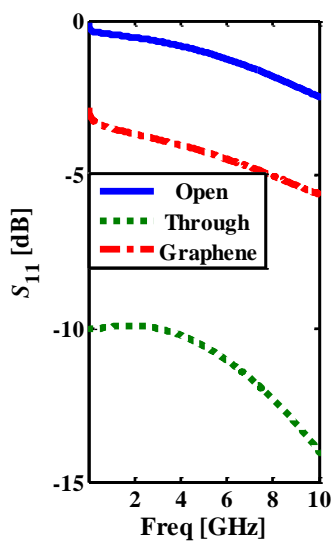

(a)

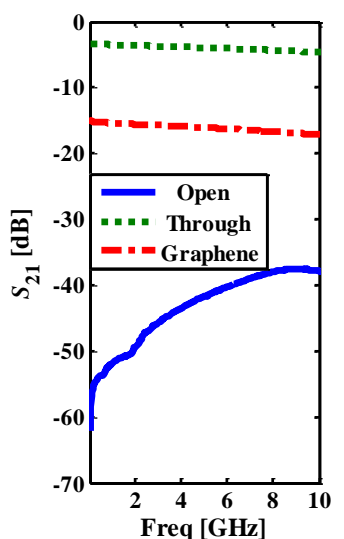

(b)
Fig. 1. (Color online) Measured reflection coefficient $S_{11}$ (a) and transmission coefficient $S_{21}$ (b) of open standard (blue solid line), through standard (green dash line) and graphene sample (red dash-dot line).

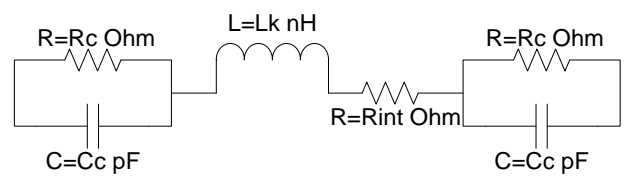

Fig. 2. Equivalent circuit model of the intrinsic graphene.

capacitance on each side of the signal trace; $R_{\text {int }}$ is the intrinsic resistance and $L_{\mathrm{k}}$ is the intrinsic inductance of the graphene sample. The corresponding fitting parameters are listed in Table I. The comparison between the experimentally obtained $S_{21}$ and the equivalent circuit model fitted $S_{21}$ is plotted in Fig. 3 , which agrees reasonably well.

Taking into account the geometry of the graphene sample (10 $\mu \mathrm{m}$ length and $25 \mu \mathrm{m}$ width), the surface resistance and surface inductance are then calculated to be $531.88 \Omega / \square$ and $0.59 \mathrm{nH} / \square$, respectively. The corresponding real surface conductivity of about $1.876 \mathrm{mS} / \square$ is comparable to reported values in literature [6-7]. It is also comparable to those reported in the $\mathrm{THz}$ regime [22-24] which enables potential $\mathrm{THz}$ antenna applications.

A statistical uncertainty analysis of the intrinsic graphene transport property is also performed based on seven $S$ parameter measurements under independent calibration conditions. Impacts of $S$-parameter uncertainties on the parameters of the equivalent circuit model in Fig. 2 are estimated using the standard deviation (SD) of the measured $S$-parameters, as listed in Table I. In summary, in the worst case scenario, the uncertainty of the graphene surface resistance and surface inductance is estimated to be \pm 57.67 $\Omega / \square$ and $\pm 0.047 \mathrm{nH} / \square$, respectively. The real and imaginary part of the graphene surface conductivity with statistical error bars are plotted in Fig. 4.

\section{B. Nonlinear Characterization}

It is theoretically predicted that graphene may possess strong electromagnetic nonlinearity because of its unique linear carrier energy dispersion. In [14], a frequency multiplier is demonstrated with a graphene sample shunting the signal trace and ground planes of a CPW line. However, power dependence effect is not studied. A microwave tripler based on microstrip structure incorporating few-layer graphene 


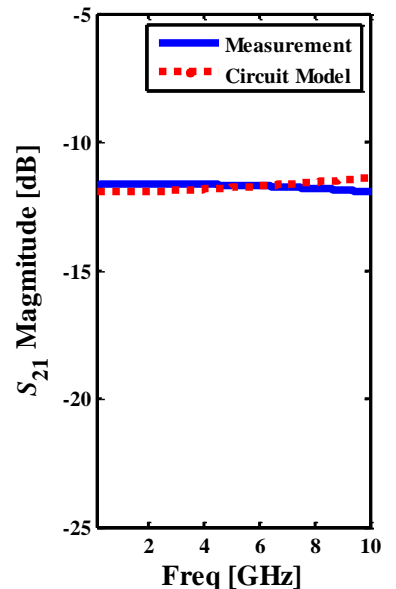

(a)

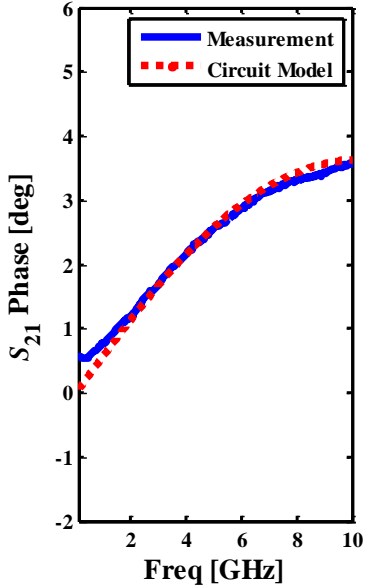

(b)
Fig. 3. (Color online) Comparison between the de-embedded experimentally obtained (average of 7 measurements, blue solid line) and the equivalent circuit model fitted (red dash line) $S_{21}$ (a) magnitude and (b) phase.

TABLE I

FItTed Parameters For The EQuivalent Circuit Model IN Fig. 2

\begin{tabular}{ccccc}
\hline \hline & $R_{\mathrm{C}}[\Omega]$ & $C_{\mathrm{C}}[\mathrm{pF}]$ & $R_{\text {int }}[\Omega]$ & $L_{\mathrm{k}}[\mathrm{nH}]$ \\
\hline $\begin{array}{c}\text { Average } \\
\text { Magnitude(S21) }\end{array}$ & 41.66 & 0.25 & 212.75 & 0.236 \\
$\begin{array}{c}\text { with positive SD } \\
\text { Magnitude(S21) }\end{array}$ & 34.35 & 0.39 & 209.74 & 0.217 \\
$\begin{array}{c}\text { with negative SD } \\
\text { Phase(S21) with } \\
\text { positive SD }\end{array}$ & 48.07 & 0.18 & 222.8 & 0.22 \\
$\begin{array}{c}\text { Phase(S21) with } \\
\text { negative SD }\end{array}$ & 37.23 & 0.31 & 235.82 & 0.253 \\
\hline \hline
\end{tabular}

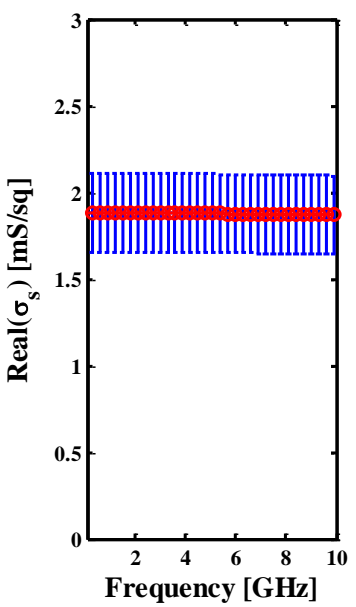

(a)

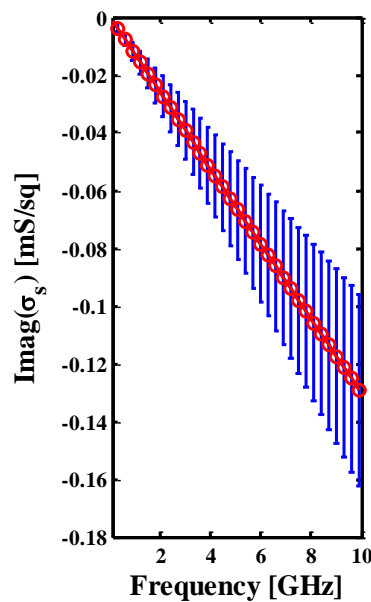

(b)
Fig. 4. (Color online) (a) Real and (b) imaginary part of the extracted graphene surface conductivity with statistical error bars based on 7 independent measurements.

exfoliated from highly oriented pyrolithic graphite is studied in [15]. In this work, the power-dependent harmonic components (second- and third-order) generated from CVD grown graphene are experimentally investigated at $1 \mathrm{GHz}$. The measurement schematic is shown in Fig. 5. Since the signal generator (SG) and the spectrum analyzer (SA) will both generate harmonics due to their intrinsic nonlinearity, the harmonic components generated in the "Through" structure are also measured at the same frequency to calibrate and eliminate those nonlinear contributions from the measurement equipment. The measured results are plotted in Fig. 6. It can

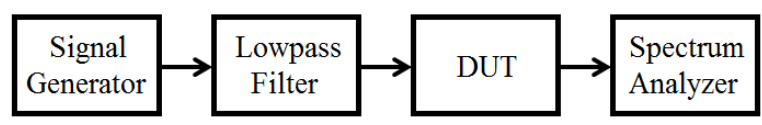

Fig. 5. Schematic of nonlinear harmonic generation measurement setup.

be seen that the third-order harmonic generated in graphene is much stronger than the second-order harmonic, as predicted by theory, while the contribution from the SG is small and that from the SA is negligible. Moreover, the second-order harmonic generated in graphene is observed to be comparable to those from the SG and SA. The slopes of the measured second-order and third-order harmonics are close to 2 and 3, respectively, as expected. In the entire power sweeping range up to $+15 \mathrm{dBm}$, there is no noticeable compression in the fundamental output (constant slope $=1$ observed).

\section{Nonlinear Effect Evaluation of a Graphene Antenna Example}

To understand the impact of nonlinear property of graphene on potential antenna applications, the measured nonlinear response is applied to a simple patch antenna similar to the one in [11] but modified to operate at $1 \mathrm{GHz}$. This patch antenna made of graphene is simulated using HFSS and has a dimension of $83.5 \mathrm{~mm}$ by $83.5 \mathrm{~mm}$. The dielectric substrate has a thickness of $1.52 \mathrm{~mm}$ and a dielectric constant of 3.2. For wireless communication applications, the two-tone thirdorder intermodulation distortion (IMD3) is one of the most important factors. From the measured third-order harmonic, the IMD3 under two-tone excitation can be easily estimated (two-tone IMD3 is a factor of 3, or $9.54 \mathrm{~dB}$ higher than the third-order harmonic). Figure 7 shows the third-order intercept (TOI) diagram of the graphene sample, indicating a TOI point of about $28 \mathrm{dBm}$ which is smaller than a typical wireless system and is due to the strong nonlinearity generation in the graphene antenna. Considering the criterion that the two-tone IMD3 should be at least $30 \mathrm{~dB}$ below the carrier signal in a wireless communication system, the maximum input power to this antenna to avoid large IMD3 is evaluated using the following approach. First, it is seen from Fig. 7 that with a fundamental input power level of $11.54 \mathrm{dBm}$ for the CPW graphene structure, the IMD3 is about $30 \mathrm{~dB}$ below the fundamental output. Second, it is assumed that the graphene patch antenna will have similar IMD3 level as the CPW graphene structure if their maximum surface current densities are at the same level. It is then estimated that the input power to the antenna should be below $22 \mathrm{dBm}$.

\section{CONCLUSIONS}

In this work, linear and nonlinear microwave properties of CVD grown graphene are characterized using a $50-\Omega$ CPW transmission line test structure with a gap at the center. The intrinsic linear transport properties of graphene are extracted from the de-embedded $S$-parameters and fitted with an equivalent circuit model. The complex surface conductivity of the graphene sample with statistical error bars is obtained as a function of frequency, showing comparable values to reported results in literature. The power dependence of second- and 


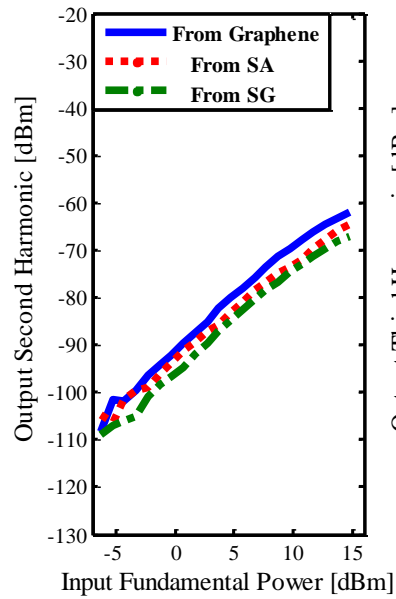

(a)

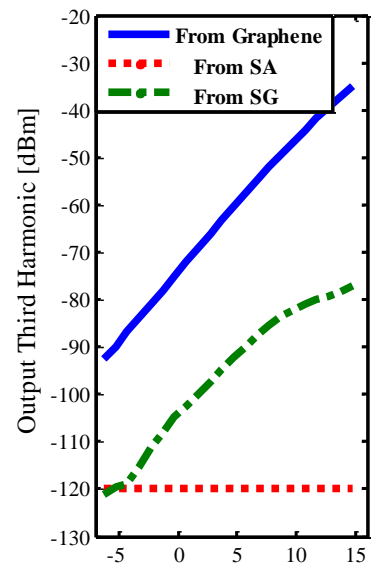

Input Fundamental Power [dBm]

(b)
Fig. 6. (Color online) Power dependence of (a) the second-order harmonic and (b) the third-order harmonic from the graphene sample and measurement equipment.

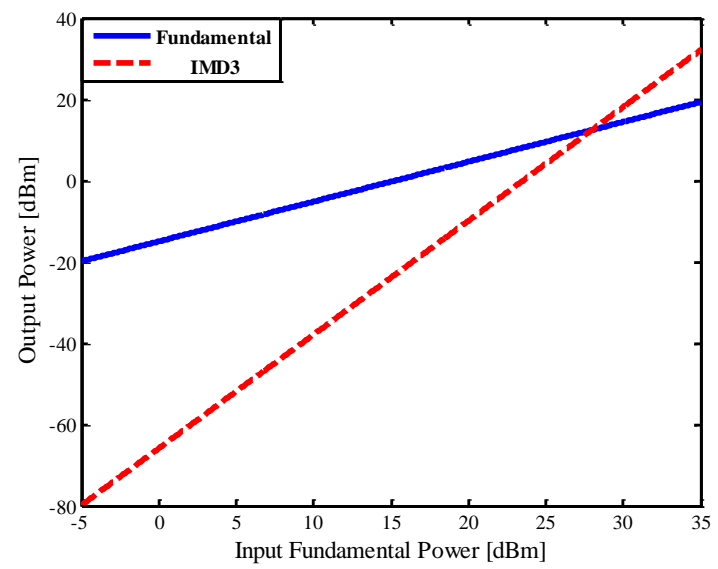

Fig. 7. (Color online) Third order intercept (TOI) of graphene sample based on nonlinearity measurement.

third-order harmonics is investigated for an input fundamental signal of $1 \mathrm{GHz}$. Strong third-order harmonic (slope of $\sim 3$ ) and weak second-order harmonic (slope of $\sim 2$ ) generation are experimentally observed. With the input power up to +16 $\mathrm{dBm}$, no power saturation is observed in the fundamental output RF power. Moreover, the measured nonlinear properties of graphene are applied to a graphene patch antenna example to evaluate its impact on potential antenna applications in terms of the IMD3 level.

\section{ACKNOWLEDGEMENTS}

We thank Prof. Krishna Muralidharan for providing the CVD setup to prepare graphene samples. Qing Hao also acknowledges the support from AFOSR (FA9550-16-1-0025).

\section{REFERENCES}

[1] A. Fallahi and J. Perruisseau-Carrier, "Manipulation of giant Faraday rotation in graphene metasurfaces," Appl. Phys. Lett., vol. 101, 231605, 2012.

[2] A. Andryieuski and A. V. Lavrinenko, "Graphene metamaterials based tunable terahertz absorber: effective surface conductivity approach," Opt. Express, vol. 21, pp. 9144-9155, 2013.

[3] B. Wu, H. M. Tuncer, A. Katsounaros, W. Wu, M. T. Cole, K. Ying, L. Zhang, W. I. Milne, and Y. Hao, "Microwave absorption and radiation from large-area multilayer CVD graphene," Carbon, vol. 77, pp. 814$822,2014$.

[4] I. Llatser, C. Kremers, A Cabellos-Aparicio, J. M. Jornet, E. Alarcón, and D. N. Chigrin, "Graphene-based nano-patch antenna for terahertz radiation," Photon. Nanostruct: Fundam. Appl., vol. 10, pp. 353-358, 2012.

[5] G. W. Hanson, A. B. Yakovlev and A. Mafi, "Excitation of discrete and continuous spectrum for a surface conductivity model of graphene," $J$. Appl. Phys., vol. 110, 114305, 2011.

[6] H.-J. Lee, E. Kim, J.-G. Yook and J. Jung, "Intrinsic characteristics of transmission line of graphenes at microwave frequencies," Appl. Phys. Lett., vol. 100, 223102, 2012.

[7] H. S. Skulason, H. V. Nguyen, A. Guermoune, V. Sridharan, M. Siaj, C. Caloz and T. Szkopek, "110 GHz measurement of large-area graphene integrated in low-loss microwave structures," Appl. Phys. Lett., vol. 99, 153504, 2011

[8] S. Moon, K. Jung, K. Park, H J. Kim, C.-W. Lee, C.-W. Baik and J. M. Kim, "Intrinsic high-frequency characteristics of graphene layers," New J. Phys., vol. 12, 113031, 2010.

[9] M. Liang, M. Tuo, S. Li, Q. Zhu and H. Xin, "Graphene conductivity characterization at microwave and $\mathrm{THz}$ frequency," 8th European Conference on Antennas and Propagation (EuCAP), pp. 489-491, April 2014.

[10] G. Vincenzi, G. Deligeorgis, F. Coccetti, and P. Pons, "Open-Thru deembedding for Graphene RF devices," IEEE Intl. Microwave Symp., pp. 1-4, June 2014.

[11] J. S. Gomez-Diaz and J. Perruisseau-Carrier, "Microwave to $\mathrm{THz}$ properties of graphene and potential antenna applications," in Proc. Int. Symp. Antennas Propag. (ISAP12), Nagoya, Japan, Nov. 2012.

[12] M. Dragoman, D. Neculoiu, A.-C. Bunea, G. Deligeorgis, M. Aldrigo, D. Vasilache, A. Dinescu, G. Konstantinidis, D. Mencarelli, L. Pierantoni and M. Modreanu, "A tunable microwave slot antenna based on graphene," Appl. Phys. Lett., vol. 106, 153101, 2015.

[13] S.A. Mikhailov, "Electromagnetic response of electrons in graphene: Non-linear effects," Physica E, vol. 40, pp. 2626-2629, 2008.

[14] M. Dragoman, D. Neculoiu, G. Deligeorgis, G. Konstantinidis, D. Dragoman, A. Cismaru, A. A. Muller and R. Plana, "Millimeter-wave generation via frequency multiplication in graphene," Appl. Phys. Lett., vol. 97, 093101, 2010.

[15] R. Camblor, S. Ver Hoeye, G. Hotopan, C. Vázquez, M. Fernández, F. Las Heras1, P. Álvarez, and R.Menéndez, "Microwave frequency tripler based on a microstrip gap with graphene," J. of Electromagn. Waves and Appl., vol. 25, pp. 1921-1929, 2011.

[16] G. R. Hotopan, S. Ver-Hoeye, C. Vazquez-Antuna, R. Camblor-Diaz, M. Fernandez-Garcia, F. Las Heras Andres, P. Alvarez, and R. Menéndez, "Millimeter wave microstrip mixer based on graphene," Prog. Electromagn. Res., vol. 118, pp. 57-69, 2011.

[17] V. Golikov, S. Hienonen, and P. Vainikainen, "Passive intermodulation distortion measurements in mobile communication antennas," in Veh. Technol. Conf., vol. 4, pp. 2623-2625, Oct. 2001.

[18] M. Tuo, L. Wang, M. R Amer, X. Yu, S. B Cronin, and H. Xin, "Microwave properties of suspended single-walled carbon nanotubes with a field-effect transistor configuration," IEEE Intl. Microwave Symp., pp. 1-4, June 2011.

[19] M. Tuo, S. Li, D. Xu, M. Liang, Q. Zhu, Q. Hao, and H. Xin, "Linear and nonlinear microwave characterization of CVD-grown graphene using CPW structure," 9th European Conference on Antennas and Propagation (EuCAP), pp. 1-2, April 2015.

[20] T. J. Gnanaprakasa, Y. Gu, S. K. Eddy, Z. Han, W. J. Beck, K. Muralidharan, and S. Raghavan, "The role of copper pretreatment on the morphology of graphene grown by chemical vapor deposition," Microelectron. Eng., vol. 131, pp. 1-7, 2015.

[21] M.-H. Cho, G.-W. Huang, K.-M. Chen, and A.-S. Peng, "A novel cascade-based de-embedding method for on-wafer microwave characterization and automatic measurement," IEEE Intl. Microwave Symp., vol. 2, pp. 1237-1240, June 2004.

[22] M. Liang, Z. Wu, L. Chen, L. Song, P. Ajayan and H. Xin, "Terahertz characterization of single-walled carbon nanotube and graphene onsubstrate thin films," IEEE Trans. on Microw. Theory and Techn., vol. 59, pp. 2719-2725, 2011.

[23] M. Liang, and $\mathrm{H}$. Xin, "Microwave to $\mathrm{THz}$ characterization of carbon based nano-materials," IEEE Microw. Mag., vol. 15, pp. 40-51, 2014.

[24] J. D. Buron, F. Pizzocchero, P. U. Jepsen, D. H. Petersen, J. M. Caridad, B. S. Jessen, T. J. Booth, and P. Bøggild, "Graphene mobility mapping," Sci. Rep., 5, 12305, 2015. 